\title{
Diversity and Co-Occurrence Patterns of microbial Communities in the Fertilized Eggs of Thitarodes Insect, the Implication on the Occurrence of Chinese cordyceps
}

\author{
Lian-Xian Guo \\ Guangdong Medical University \\ Xiao-Shan Liu \\ Guangdong Medical University \\ Zhan-Hua Mai \\ Guangdong Medical University \\ Yue-Hui Hong \\ Guangdong Hospital of Traditional Chinese Medicine \\ Qi-Jiong Zhu \\ Guangdong Medical University \\ Xu-Ming Guo \\ Guangdong Medical University \\ Huan-Wen Tang ( $\nabla$ thw@gdmu.edu.cn ) \\ Guangdong Medical University
}

\section{Research article}

Keywords: Bacterial, Fungal, Fertilized Eggs, Thitarodes, Chinese cordyceps, Wolbachia

Posted Date: April 28th, 2020

DOI: https://doi.org/10.21203/rs.3.rs-24686/v1

License: (c) (i) This work is licensed under a Creative Commons Attribution 4.0 International License. Read Full License 


\section{Abstract}

Background The large-scale artificial cultivation of Chinese cordyceps has not been widely implemented because the crucial factors triggering the occurrence of Chinese cordyceps have not been fully illuminated. Methods In this study, the bacterial and fungal structure of fertilized eggs in the host Thitarodes collected from 3 sampling sites with different occurrence rates of Chinese cordyceps (Sites A, B and C: high, low and null Chinese cordyceps, respectively) were analyzed by performing 16S RNA and ITS sequencing, respectively. And the intra-kingdom and inter-kingdom network were analyzed. Results For bacterial community, totally 4671 bacterial OTUs were obtained. a-diversity analysis revealed that the evenness of the eggs from site A was significantly higher than that of sites $B$ and $C$, and the dominance index of site $A$ was significantly lower than that of sites $B$ and $C(P<0.05)$. $\beta$-diversity analysis showed that the differences of bacterial community among the eggs from the three sampling sites were significantly different. OTU1 (Wolbachia) was the overwhelming predominant bacteria in the eggs from sites B and C. Although OTU4 (Spiroplasma) was detected in minor abundances, it showed distribution preference in the fertilized eggs from site A. For fungal community, totally 3318 fungal OTUs were obtained. Difference analysis showed significant differences among the three sites, while the differences were not as significant as that of bacterial community. In addition, ten fungal genera in the three most concerned Cordyceps families (Clavicipitaceae, Cordycipitaceae and Ophiocordycipitaceae) were detected in the fertilized eggs, while the most approved anamorph ( Hirsutella ) of Chinese cordyceps wasn't discovered. Intra-kingdom (fungi) network analysis revealed more positive correlations and average degrees at sites A, and Inter-kingdom network analysis revealed more positive and negative correlations at sites A. Discussion The microbial community, especially the bacterial community in the fertilized eggs, might be significantly related with the occurrence of Chinese cordyceps, and Wolbachia might be the most significant microorganism negatively related with the occurrence. A closer correlation of the microbial community, especially closer fungal positive correlation, in the fertilized eggs might help for the occurrence of Chinese cordyceps.

\section{Background}

Ophiocordyceps sinensis is a famous fungus-larva complexity indigenously grown in the Qinghai-Tibetan Plateau and its surrounding high-altitude regions [1-2]. O. sinensis obligately infects and parasitizes the Thitarodes (Hepialidae, Lepidoptera) larva [3] and ultimately forms fungus-caterpillar complex, which is commonly nominated as Chinese cordyceps [1]. In this paper, in order to avoid misunderstanding, Chinese cordyceps and $\boldsymbol{O}$. sinensis were used to refer to the fungus-larva combination and the entomogenous fungus, respectively. It has enjoyed high confidence and popularity owing to the functions in treating diverse diseases as a traditional medicinal herb for thousands of years in oriental countries, particularly in China [4]. The distinguished medicinal efficiency stimulated a large demand for natural Chinese cordyceps, while the yield is extremely limited by the reason of its complex life history [1,5], obligatory parasitism [6] and eco-geographical uniqueness [7]. Even worse, in recent years, the population of natural Chinese cordyceps sharply declined owing to excessive excavation, habitat destruction and global warming [8]. The imbalance between supply and demand resulted in the significant rise of its trading price [9], and accordingly aggravating the excavation. Scientists have striven for the large-scale cultivation of Chinese cordyceps in recent decades, and achieved many progresses. However, the commercially artificial cultivation has not been widely implemented because the 
key factor(s) for triggering the occurrence of Chinese cordyceps (germination of stromata from the stiff larvae) has (have) not been confirmed [10].

The infection of the host Thitarodes larva usually happens in habitat soils (Fig. 1a). With the rainfall, the fungal spores erupted from the mature stroma of Chinese cordyceps gradually infiltrate into soil, and develop to infective conidia to infect the susceptible Thitarodes larva [11]. Besides $O$. sinensis, our previous study also revealed that several physicochemical factors, the soil bacterial and fungal structure, and the network of these factors, are closely related with the occurrence of Chinese cordyceps, manifesting that the soil ecological environment is important for the occurrence of Chinese cordyceps [12]. Observation on the indoor cultivated Thitarodes larva revealed that, although collected from the same sampling site, the fates of these wild larvae varied greatly and a proportion of these larvae eventually formed Chinese cordyceps [13]. Thus, we proposed that, besides external soil factors, the internal factors of the Thitarodes host (such as the entophytic microbes and immune system of Thitarodes) may also contribute to occurrence of Chinese cordyceps.

To investigate the internal factors relevant with the occurrence of Chinese cordyceps, in our subsequent study, we innovatively analyzed the microbial diversity and community of the unfertilized eggs mass of the Thitarodes moth [14]. However, in that study, the analysis on a-diversity, $\beta$-diversity, and the microbial structure revealed no significant differences among the groups with different potential occurrence rates of Chinese cordyceps. Furthermore, the revealed microbes in that study were actually originated from both the unfertilized eggs and the adhesive ovary tissues of mother moths, which were difficult to be separated off. To prevent the disturbance from the ovary tissues of mother moths, and investigate the influence from the mating behavior, in this study, we chose the laid-out eggs (fertilized eggs) to perform $16 \mathrm{~S}$ and ITS sequencing. The microbial factors correlated with the occurrence of Chinese cordyceps were screened out (such as Wolbachia and Spiroplasma) and further discussed. Our new findings may provide a more distinct and comprehensive clue for further illuminating the relationship between fungi and their host and uncovering the occurrence of Chinese cordyceps.

\section{Results}

\section{Microbial Diversity}

Through the raw sequencing reads, there was a total of 1379003 high-quality 16S rDNA sequences and 686849 high-quality ITS2 sequences. Accordingly, 4671 bacterial OTUs and 3318 fungal OTUs (supplemental table) were clustered from these high-quality sequences with a $97 \%$ identity threshold. The number of bacterial sequences of site $\mathrm{C}$ was significantly higher than that of sites $\mathrm{A}$ and $\mathrm{B}$. a-diversity indices of bacterial and fungal communities are shown in Table 1. The evenness (represented by Simpson and Shannon indices) of bacterial diversity of site A was significantly higher than that of sites $\mathrm{B}$ and $\mathrm{C}(P<0.05)$, and the dominance index of site A was significantly lower than that of sites $B$ and $C(P<0.05)$. For fungi, the indices of PD whole tree and Dominance of site $C$ were significantly higher, and the Simpson index of site $C$ was significantly lower than that of site $\mathrm{B}(P<0.05)$. Venn diagrams (Fig. 1b and c) illustrated that 39 bacterial OTUs and 41 fungal OTUs were shared among all of the detected eggs samples (the detailed taxonomic information was supplemented in supplemental table) and were considered as core microbiome [15].

Beta-diversity at the OTU level by NMDS analysis is shown in Fig. 1d and e. For bacteria, the dots from sites A and $\mathrm{B}$ were distributed dispersedly while the dots from site $\mathrm{C}$ were grouped closely. In addition, the dots from 
site A were obviously far from sites B and C, and the samples within each site were not situated closely. For fungi, the samples from all of the three sites distributed dispersedly. Furthermore, significant differences $(P<$ 0.05 ) were found among all of the samples in aspects of bacterial and fungal communities, as shown in Table 2, corresponding to ANOSIM, Adonis, and MRPP analysis. Specifically, the R for ANOSIM analysis of bacteria is 0.6053 ; $F$ and $R^{2}$ for Adonis analysis of bacteria is 19.342 and 0.76324 , respectively; and the effect size (A) of bacteria for MRPP analysis is 0.476 , indicating that the difference among groups of bacterial community is larger than the difference within groups. For fungal community, R for ANOSIM analysis and the effect size (A) for MRPP analysis is 0.2702 and 0.06414 , respectively, which approach 0 , indicating that although the differences among the groups are significant $(P<0.05)$, they are less significant than that of bacterial community.

\section{Bacterial and Fungal Structure}

Bacterial and fungal construction (relative abundances) at the phylum, class, order, family, genus level, and OTU table are supplemented in supplemental table, respectively. Based on these data, the top 20 phyla and top 20 families of bacterial and fungal communities were illustrated using histograms in Figs. 2a-d, and the top 40 OTUs were illustrated using heat map in Figs. 2e and f.

For bacterial community, the microbial structures displayed significantly different patterns among different sampling sites both at phylum (Fig. 2a), family (Fig. 2c) and OTU (Fig. 2e) rank, especially between site A and site B/C. At phylum rank, for the samples from site A, Firmicutes (40.1\%-57.2\%), Proteobacteria $(26.2 \%-33.5 \%)$, and Actinobacteria (8.3\%-15.4\%) were the major phyla. For sites B and C, Proteobacteria ranked the overwhelmingly predominant phyla, with the proportion range of $81.0 \%-97.2 \%$ and $78.2 \%-95.0 \%$ in $B$ and $C$, respectively. The situation in site $\mathrm{C}$ was slightly different from site $\mathrm{B}$. Besides Proteobacteria, Firmicutes was with a noticeable proportion (3.87\%-19.8\%). At family rank, in site A, Unclassfied_66 (22.0\%-51.6\%), Moraxellaceae (8.8\%-15.2\%) and Staphylococcaceae ( $0.63 \%-9.78 \%)$ were the top 3 families. In sites B and C, Anaplasmataceae was the uppermost family, with the relative proportions of $72.6 \%-96.6 \%$ and $73.8 \%-93.2 \%$, respectively. At OTU level, Fig. 2e presented distinct patterns among the three sampling sites, showing that Wolbachia (OTU_1 and OTU_24871) was the overwhelmingly predominant OTU in sites B and C, while the other 38 OTUs were minor in the two sites. Conversely, Wolbachia (OTU_1 and OTU_24871) was in trace abundance while the other 38 OTUs were abundant and distributed evenly in site A. OTU_2 (Firmicutes), OTU_7 (Acinetobacter), OTU_9 (Staphylococcus), OTU_10 (Deinococcus), and OTU_12 (Enhydrobacter) were the top 5 OTUs in the egg samples at site $A$. The predominant OTUs at sites $B$ and $C$ were distinct with that at site $A$, with OTU_1 (Wolbachia), OTU_2 (Firmicutes), OTU_6 (Carnobacterium), OTU_24871 (Wolbachia), and OTU_29 (Pseudomonas) ranking the top 5 OTUs in the egg samples at site B, and OTU_1 (Wolbachia), OTU_2 (Firmicutes), OTU_7 (Acinetobacter), OTU_24871 (Wolbachia), and OTU_13 (Cupriavidus) ranking the top 5 OTUs in the egg samples at site $\mathrm{C}$. Conclusively, the bacterial composition at different levels revealed distinct differences among different sites, especially the differences between high Chinese cordyceps group (site A) and the low/null Chinese cordyceps group (site B/C).

For the fungal community (Figs. $2 \mathrm{~b}$ and d), the differences of the predominant fungi among different groups were not as remarkable as that of the bacteria. In addition, different from bacterial composition, the fungal composition at phylum and family levels from site B presented more distinction with that from sites $A$ and $C$. At phylum level, Ascomycota, unclassified fungi, and Basidiomycota were the top 3 predominant fungal phyla. 
Among them, Ascomycota was the most predominant in 7 fertilized eggs samples (in E-A2, E-B1, E-B2, E-B3, EB4, E-B5, and E-C2, and ranged from 51.7\%-82.2\%). In the remaining 8 unfertilized egg samples (in E-A1, E-A3, EA4, E-A5, E-C1, E-C3, E-C4, and E-C5, and ranged from 5.9\%-38.4\%), the unclassified fungi were overwhelmingly predominant. Basidiomycota was the third abundant fungi (ranged in 5.8\%-20.9\%) in all of the detected samples. At family rank (Fig. 2d), in site A, Unclassfied_2, Trichocomaceae, Cladosporiaceae, Unclassfied_21 and Sclerotiniaceae were the top 5 families; in site B, Trichocomaceae, Unclassfied_2, Unclassfied_3, Parmeliaceae, and Filobasidiaceae were the top 5 families; and in site C, Unclassfied_2, Trichocomaceae, Unclassfied_21, Unclassfied_3, and Unclassfied_22 were the top 5 families. In addition, Fig. 2d illustrated more stable distribution within group $\mathrm{C}$, while more variable distributions were observed within groups $\mathrm{A}$ and $\mathrm{B}$. Figure $2 \mathrm{f}$ illustrated distinct heat map patterns among the three sites. At site A, OTU_4, OTU_8 (Cladosporium cladosporioides), OTU_6 (Penicillium polonicum), OTU_7 (Penicillium) and OTU_10 were the top 5 OTUs; OTU_6 (Penicillium polonicum), OTU_12 (Bryoria), OTU_8 (Cladosporium cladosporioides), OTU_10 (Aspergillus conicus) and OTU_22 (Ascomycota) were the top 5 OTUs at site B; and OTU_4, OTU_7, OTU_6 (Penicillium polonicum), OTU_15 and OTU_19 were the top 5 OTUs at site C.

In terms of the most concerned Cordyceps-related families [16], Clavicipitaceae, Cordycipitaceae, and Ophiocordycipitaceae were identified but in minor abundance in this study (Fig. 3a). In general, the Cordycepsrelated families displayed preference at sites B and C. At genus level, Purpureocillium showed distribution preference at site A. Engyodontium, Lecanicillium, Pochonia, Simplicillium, Cordyceps, and Chaunopycnis showed distribution preference at site B. Metarhizium and two other genera belonging to Clavicipitaceae and Cordycipitaceae showed distribution preference at site $\mathrm{C}$.

\section{Differential OTUs among Different Sampling Sites}

In order to discuss the detailed OTUs that might be related to the occurrence of Chinese cordyceps, the differential OTUs among different sites were screened out as biomarkers using LDA effect size analysis (Fig. 4). Fifty-four bacterial OTUs (belonging to orders of Actinobacteria, Flavobacterlia, Deinococci, Bacilli,

Betaproteobacteria, and Gammaproteobacteria) and ten fungal OTUs presented significantly higher abundance in the samples of the high Chinese cordyceps group (site A). Five bacterial OTUs and thirty-five fungal OTUs presented significantly higher abundance in the samples of the low Chinese cordyceps group (site B). Twelve fungal OTUs presented significantly higher abundance in the samples of the null Chinese cordyceps group (site C).

\section{Co-Occurrence Analysis}

The intra-kingdom network analysis were conducted based on the top 40 bacterial and fungal genera (Table 3 and Fig. 5). For bacterial analysis (Figs. 5a,c,e), 89 positive and 36 negative, 237 positive and 37 negative, and 97 positive and 33 negative significant correlations were observed at sites $A, B$, and $C$, respectively. For fungal analysis (Figs. 5b,d,f), 114 positive and 28 negative, 43 positive and 52 negative, and 57 positive and 27 negative significant correlations were observed at sites $A, B$, and $C$, respectively. The average degree of bacterial communities was $4.3,11.7$ and 4.5 , and fungal communities was $6.6,4.8$ and 4.2 , respectively. The interkingdom network analysis were conducted among all of the bacterial and fungal families. Among all of the detected bacterial and fungal families, highly significant positive and negative correlations (Spearman) were extracted and the related network analysis are summarized in Table 3 and visualized in Fig. 6. Briefly, in group A, B and C, there were 593, 386, and 116 links in total, respectively, with 198, 139 and 44 positive significant 
correlations, and 395, 247 and 72 negative significant correlations, respectively. The amount of the nodes was 46,40 and 20 , and the average degree was $25.8,19.3$ and 11.6, respectively. Thus, Inter-kingdom connections and relationships in the microbial community were observed the most and the closest in group $\mathrm{A}$, and gradually reduced and alienated in group $\mathrm{B}$ and group $\mathrm{C}$.

\section{Discussion}

Fertilization Remodels the Microbial Community of the Thitarodes Eggs. From the years of 2006 to 2016, we had conducted a pilot investigation at Shergyla mountain to survey the density of Chinese cordyceps and host Thitarodes larvae [12], and ultimately had screened out three sampling sites (see 2.1) with different occurrence rates for our series of studies. To study the influence from environmental microbes on the occurrence of Chinese cordyceps, we had comparatively analyzed the external (soil) and internal microbial community on Thitarodes insects [12,14]. For external soil habitat, both the bacterial and fungal community varied significantly among the three sampling sites [12]; for internal microbial analysis, we chose the unfertilized eggs in female Thitarodes moths as research sample, and found significant differences $(p<0.05$, ANOSIM, Adonis, and MRPP analyses) of bacterial community among the three sites, while no significant differences were observed $(p>0.05)$ of fungal community [14]. In this study, the laid out fertilized eggs were analyzed. It's surprising to find significant differences $(P<0.05)$ among the three sites in aspects of both bacterial and fungal communities (Table 2). Furthermore, unlike unfertilized eggs, $a$ - and $\beta$-fungal diversities and the microbial composition of bacteria in the fertilized eggs varied regularly among different sites, which were totally different from that in unfertilized eggs. According to the life habit of Thitarodes, the Thitarodes moths mate immediately after eclosion from the pupae. During the mating process which lasts for 2.5 hours on average, semen ejected from the male Thitarodes moth is stored in the bursa copulatrix of the female, which is located near the ovipositor [17]. After the male Thitarodes leaves, the female moth begins to lay eggs to the ground, and these eggs are simultaneously fertilized with the stored semen through the micropyle [18]. To avoid the disturbance from the external habitat, we collected the fertilized eggs in asepsis condition (see 2.1). Thus, it can be inferred that fertilization may remodel the microbiome in the eggs. Nevertheless, another possibility is that the ovarian tissue firmly attached on the unfertilized eggs might be the reason for the irregular variety of the microbial community [14], which cannot be ignored.

The Microbiome in the Fertilized Eggs might Be Related with the Occurrence of Chinese cordyceps. Some intrinsic bacterial factors might be significant in the occurrence of Chinese cordyceps. Controversy has surrounded the anamorphs of $O$. sinensis since the 1980s. Currently, at least 37 genera and 90 fungal species related to the anamorphs of $O$. sinensis have been isolated [19-20]. Among these fungi, Hirsutella sinensis is the most widely accepted anamorph [21]. However, the $H$. sinensis strain isolated from the stroma of Chinese cordyceps could not induce the germination of stroma on healthy Thitarodes larvae in the laboratory [22]. Therefore, according to Koch's postulates, the pathogenicity of $H$. sinensis has not been proven. Recently, scientists proposed that Chinese cordyceps constitutes a complex and ordered microecological system instead of being a solitary organism, and hence, sole $H$. sinensis could not trigger the occurrence of Chinese cordyceps, which might be more likely induced by the synergy among $H$. sinensis and some other microorganim(s) [23]. Consistent with this inference, studies have shown that the synergy between $\mathrm{H}$. sinensis and other related fungi, such as Paecilomyces hepiali and Aspergillus tennesseensis, enhances the potency of infection [24]. Among the reported fungal combinations, different combinations promote the formation of stiff worms to varying degrees, 
while stromata also do not germinate successfully. Thus, the definite microbial factors inducing the occurrence of Chinese cordyceps remain to be investigated.

Due to the long and complex life cycles of the larvae, systematic research on the colonized microorganisms of Thitarodes larvae is difficult to conduct. Furthermore, the intrinsic bacterial factors present in Thitarodes larvae are complex and vary with changes in the soil environment [7], which contains highly abundant microbial biomass [12]. To exclude interference from the soil environment, this study chose fertilized eggs as research sample and found that fertilization remodeled the microbial community of the eggs, and the microbial factors especially bacterial factors might play a more significant role in the occurrence of Chinese cordyceps, owing to the more significant differences revealed by a-diversity and $\beta$-diversity analyses $(P<0.05)$ among different groups. For the bacterial microbiome at site A (high Chinese cordyceps group), bacterial a-diversity was significantly higher than that of sites B (low Chinese cordyceps group) and C (null Chinese cordyceps group) indicated by Shannon and Simpson indices; 20 top families evenly distributed without special bias which occurred in sites $B$ and $C$, and LEfSe analyses revealed much abundant biomarkers which specially enriched in site $A$ than that of sites B and C. Thus, the bacterial community in fertilized Thitarodes eggs might be significant for the occurrence of Chinese cordyceps, and the high evenness of the community might aid for the occurrence of Chinese cordyceps.

In each microhabitat, microbial species is not an independent existence, and it lives in a complicated community with interactions among the other microbial species. These interactions mainly include positive (commensalism, mutualism) and negative (amensalism, parasitism or predation, and competition) relation [25]. Network analysis was applied to investigate these potential microbial interactions in the complex microbial communities [26]. Chinese cordyceps is essentially the outcome of the competition of the entomogenous fungus $(H$. sinensis) and the other microbial inhabitants in the host insects [13]. Thus, the microbial interactions would influence the infection, colonization, and growth of $H$. sinensis, which ultimately related with the occurrence of Chinese cordyceps. Co-occurrence patterns were explored to offer insight into microbial interactions. According to the intra-kingdom network analysis based on the positive and negative correlations among the 40 most abundant fungal genera (Table 3 and Fig. 5), there were more positive correlations and average degrees in the high Chinese cordyceps group (sites A). Furthermore, according to Inter-kingdom network analysis based on the positive and negative correlations among the different bacterial and fungal families, the number of positive correlations, negative correlations and the total links for sites A were also the highest (Table 3 and Fig. 6). These findings indicate that a closer correlation of the microbial community, especially closer fungal positive correlation, in the fertilized eggs might help for the colonization of $O$. sinensis. This discovery reinforced the above-mentioned theory that the synergy among $H$. sinensis and some other microorganim(s) which ultimately triggers the occurrence of Chinese cordyceps [23].

Wolbachia and Spiroplasma might Be Significantly Related with the Occurrence of Chinese cordyceps. In our previous study, we found Cordyceps-related fungi (10 genera in total) may enter the host Thitarodes as early as in the oocyte stage. Among them, Purpureocillium, which also belongs to the Ophiocordycipitaceae family [27], was detected in unfertilized egg samples from the Chinese cordyceps group, providing the possibility of maternal infection and enlarging the knowledge on the infection mechanism [23]. In addition, Purpureocillium was supposed to manipulate the behaviors of its host insect Edessa rufomarginata [28]. This interesting phenomenon bears a striking resemblance to the infected Thitarodes larvae. In this study, totally 10 genera of 
Cordyceps-related fungi were observed in the fertilized eggs. Coincidently, Purpureocillium also present preference in site A. The similar distribution presence of Purpureocillium in both unfertilized [14] and fertilized eggs from site A reinforced our previous inference that this fungus in Ophiocordycipitaceae family might be in assistance for the occurrence of Chinese cordyceps. More interestingly, as the anamorph of Chinsese cordyceps, $H$. sinensis hasn't been detected in the soils [12], unfertilized [14] or fertilized eggs. The absence revealed that, $H$. sinensis, at least during the egg stage, is not predominant in the Thitarodes insect. And it might be from the result that it win the battle against the immune system of Thitarodes insect at laval stage, and ultimatley kill the host and become the biggest overlord of the whole microbiome. In addition, based on our investigations on the soil, unfertilized eggs and fertilized eggs, the Cordyceps-related fungi are in trace abundance in these samples. These results reinforced the discovery of Zhong, which revealed that most of the entomogenous fungi, including Cordyceps-related fungus and $H$. sinensis, prefer to colonize in plant roots and derive nutrition from plant sources in the absence of insect hosts [29]. While after uptake into the insect host (Thitarodes larvae) via feeding behaviors, for certain opportunity, $H$. sinensis would seize the opportunity.

Spiroplasma might aid for the occurrence of Chinese cordyceps. We had found that OTU4 (Spiroplasma) preferred to exist in the unfertilized egg samples of site A and was the dominant bacteria in some samples of site A [14] (Fig. 3b). While in the subsequent fertilized eggs, the abundance of OTU4 was very low (even could not ranked in top 40 OTUs (Fig. 2e), indicating that OTU4 may originated from the ovarian tissue of the mother which attached on the unfertilized eggs, and only a small part can enter into the fertilized eggs. Nevertheless, it still shows preference in site A and absent in sites B and C (Fig. 3c). Thus, Spiroplasma might play positive roles in the occurrence of Chinese cordyceps in the subsequent insect stages. However, the inference was based on the correlation between the abundance of Spiroplasma and potential Chinese cordyceps occurrence, and need to be verified by further experimental studies.

Wolbachia might be the inhibitory bacterium for the occurrence of Chinese cordyceps. In unfertilized eggs, OTU1 was the predominant bacterium and presented distribution preference in site B (predominant in 4 of 5 samples of site $B$, Fig. $3 b$ ), and occurred in minor amount in all of the rest samples including samples from sites $A$ and $C$ [14]. While in fertilized eggs, OTU1 ranked the overwhelming predominance in all of the samples from sites $B$ and C. Interestingly, it was absent in the samples from site A (Fig. 3c). This study revealed that the mating behaviors would also introduce the Wolbachia to the fertilized eggs, owing to the distinct difference between the abundance in the eggs from site $\mathrm{C}$ before (in minor abundance) and after (the most predominant) fertilization. In contrast to site $\mathrm{C}$, Wolbachia was detected in minor abundances in the eggs before fertilization at site $A$, while it was absent in the fertilized eggs at site $A$, the decrease might be caused by the inhibition from the Spiroplasma [30]. The strict distribution preference in low (site B) and null Chinese cordyceps group (site C) indicated that Wolbachia might play negative roles in the occurrence of Chinese cordyceps. Wolbachia is an intracellular Gram-negative bacterial genus that infects a vast range of arthropod species, probably making it the most prevalent endosymbiont in the world. Wolbachia has evolved the ability to cause reproductive alterations in its arthropod hosts, such as cytoplasmic incompatibility, parthenogenesis, feminization, and male killing [31]. More recently, Wolbachia has attracted concerns for its ability in increasing host fecundity, and immunity enhancement to protect against pathogens [32-33]. By coincidence, this study revealed that, except the predominant Wolbachia, the other bacteria are in minor abundance in sites $\mathrm{B}$ and $\mathrm{C}$ and the discovery reinforced the pathogen inhibition effect from Wolbachia. While in site A, without the inhibition from Wolbachia, the bacterial diversity and evenness are much higher (Table 1, Fig. 2e and 3c). It can be inferred that, in the 
subsequent larval stage, the growth and colonization of multiple bacteria create an appropriate living environment for the survive of $H$. sinensis, and ultimately induced the invasion and colonization of fungi $H$. sinensis, which triggers the occurrence of Chinese cordyceps.

\section{Conclusions}

The current study revealed that the microbial community especially the bacterial community was significantly related with the occurrence of Chinese cordyceps. Network analysis indicated that a closer correlation of the microbial community, especially closer fungal positive correlation, in the fertilized eggs might help for the occurrence of Chinese cordyceps. Among all of the detected microbes, Wolbachia might play negative roles, while Spiroplasma and Purpureocillium might play positive roles in the occurrence of Chinese cordyceps. Thus, the current study would provide significant information for illuminating the occurrence of Chinese cordyceps which might provide guidance for the large-scale cultivation. Nevertheless, all of these inferences are deduced based on the 16S rRNA and ITS sequencing, and need to be verified through multi-strain infection trail in future work.

\section{Methods}

\section{Study design}

Firstly, five pairs of mating Thitarodes adults were collected at three sampling sites with different occurrence of Chinese cordyceps. Secondly, the fertilized Thitarodes were obtained under aseptic condition. Thirdly, the bacterial and fungal community in the fertilized were determined using 16S rDNA and the ITS sequencing, respectively. Based on the results, the microbial factors correlated with the occurrence of Chinese cordyceps were discussed.

\section{Sampling}

The study region of the native habitats of Chinese cordyceps was situated at Shergyla Mountain, Tibet. From the years of 2006 to 2016, a pilot investigation was conducted to survey the density of Chinese cordyceps and host Thitarodes larvae [12]. Field observation showed that the peaks of activity, growth, feeding, development, and population density of Thitarodes larvae often occurs between June and August, particularly, around midJuly. Three sampling sites (site A: $29^{\circ} 36^{\prime} 09.6^{\prime \prime} \mathrm{N}, 9^{\circ} 36^{\prime} 23.1^{\prime \prime}$; site B: $29^{\circ} 35^{\prime} 49.6^{\prime \prime} \mathrm{N}, 94^{\circ} 36^{\prime} 12.0^{\prime \prime} \mathrm{E}$; and site C: $29^{\circ} 36^{\prime} 11.0^{\prime \prime} \mathrm{N}, 94 \circ 36^{\prime} 19.0^{\prime \prime} \mathrm{E}$ ) with high (site A: 50 larvae/ $\mathrm{m}^{2}, 5$ Chinese cordyceps $/ \mathrm{m}^{2}$, occurrence rate $10.0 \%$ ), low (site B: 70 larvae $/ \mathrm{m}^{2}, 1$ Chinese cordyceps $/ \mathrm{m}^{2}$, occurrence rate $0.4 \%$ ), and null (site C: 75 larvae $/ \mathrm{m}^{2}, 0$ Chinese cordyceps $/ \mathrm{m}^{2}$, occurrence rate 0 ) occurrence rate of Chinese cordyceps were ultimately selected for this study in the middle of July, 2016 [12].

To exclude the disturbance of external environmental microbes, sampling was performed as follows. At each site, five pairs of the mating moths of Thitarodes pui [34] were collected in the twilight, and each pair was put in a sterilized plant tissue culture flask $(250 \mathrm{~mL})$ and brought to the nearby laboratory. In the laboratory, the sampling flasks were situated and observed under the sterile condition. After the male moth departed, the female moth began to lay eggs immediately. Approximately 500 grains of eggs from each female were obtained, and the transverse diameter of eggs were around $0.5 \mathrm{~mm}$. The initially laid-out eggs were white and 
soft, ten hours later, they would turn into black with hard surface shell. These black eggs were carefully collected, named as A1-A5, B1-B5 and C1-C5 according to the mating moths from sampling sites, washed with sterile water for three times, and then stored at $-20^{\circ} \mathrm{C}$ before DNA extraction.

\section{DNA Extraction, PCR, Library Preparation, Sequencing, and Data Analysis}

The following procedures were conducted according to our recent study for the synchronously collected soil samples [12]. Briefly, the total DNA of the eggs were extracted and purified with the MO BIO PowerSoil@ DNA Isolation Kit (MO BIO Laboratories Inc., Carlsbad, CA, USA), with sterile water as negative control. The quality of the products were assessed via the NanoD rop ND-3300 spectrophotometer (NanoDrop Technologies, Thermo Scientific, Wilmington, DE, USA).

The $\mathrm{V} 4$ region of bacterial $16 \mathrm{~S}$ rDNA and the ITS2 region of the fungal ITS gene were targeted for amplification. $515 F / 806 \mathrm{R}$ and ITS3/ITS4 were chosen as the primers for bacterial and fungal fragments, respectively, and each primer was added with a unique 12-bp barcode sequence at the 5 -end to discriminate each sample. The PCR reaction mixture $(50 \mu \mathrm{L})$ contained Ex Taq DNA polymerase ( 0.5 units; TaKaRa, Dalian, China), $1 \times$ Ex Taq loading buffer (10 $\mu \mathrm{L}$; TaKaRa, Dalian, China), dNTPs ( $8 \mu \mathrm{L}$; TaKaRa, Dalian, China), $2 \mu \mathrm{L}$ of each primer $(10 \mathrm{mM})$, and DNA template (10-100 ng). PCR was conducted through the ABI GeneAmp ${ }^{\circledR} 9700$ PCR System (Applied Biosystems, Waltham, MA, USA) using the following conditions for bacterial-specific fragments: $95^{\circ} \mathrm{C}$ for $3 \mathrm{~min} ; 35$ cycles of $94^{\circ} \mathrm{C}$ for $30 \mathrm{~s}, 55^{\circ} \mathrm{C}$ for $1 \mathrm{~min}$, and $72{ }^{\circ} \mathrm{C}$ for $1 \mathrm{~min}$; and $72{ }^{\circ} \mathrm{C}$ for $10 \mathrm{~min}$. For fungalspecific fragments, the PCR procedures included $95^{\circ} \mathrm{C}$ for $5 \mathrm{~min} ; 30$ cycles of $95^{\circ} \mathrm{C}$ for $30 \mathrm{~s}, 52{ }^{\circ} \mathrm{C}$ for $30 \mathrm{~s}$, and $72{ }^{\circ} \mathrm{C}$ for $45 \mathrm{~s}$; and final extension at $72{ }^{\circ} \mathrm{C}$ for $10 \mathrm{~min}$. Each sample was carried out with triplicate PCR reactions, and these PCR products were mixed and purified with EZNA Gel Extraction Kit (Omega, USA).

Sequencing libraries were created through NEBNext ${ }^{\circledR}$ Ultra ${ }^{\text {TM }}$ DNA Library Prep Kit for Illumina ${ }^{\circledR}$ (New England Biolabs, MA, USA) according to the manufacturer's recommendations, and index codes were added. After the assessment on the Qubit 2.0 Fluorometer (Thermo Fisher Scientific, MA, USA) and Agilent Bioanalyzer 2100 system (Agilent Technologies, Waldbron, Germany), the libraries were sequenced via an Illumina MiSeq PE300 platform at Ozimeks Biotech Co., Ltd. (Shenzhen, Guangdong, China). 250 bp paired-end raw reads were generated and then filtered according to the Trimmomatic [35] (V0.33, http://www.usadellab.org/cms/? page=trimmomatic). Subsequently, the paired-end clean reads were merged using FLASH [36] (V1.2.11, https://ccb.jhu.edu/software/FLASH/) according to the overlap between the paired-end reads, and the merged sequences were assigned to each sample according to the unique barcode using Mothur software [37] (V1.35.1, http://www.mothur.org). The obtained sequence data were deposited in the Sequence Read Archive (PRJNA587795). Sequence analysis was carried out by USEARCH software [38] (V10, http://www.drive5.com/usearch/), and $97 \%$ similarity threshold was set for the assignment of the same operational taxonomic units (OTUs) [39]. The sequence with the most frequency was extracted as the representative sequence for each OTU for taxonomic annotation.

The SILVA (for 16S, v. 119; http://www.arb-silva.de) and Unite (for ITS, v. 7.0; http://unite.ut.ee/index.php) databases were chosen for bacterial and fungal taxonomic annotation, respectively (the confidence threshold default $\geq 0.5$ ). The OTU and its Tags annotated as chloroplasts or mitochondria (16S amplicons) or can't 
annotated to the kingdom rank were filtered. The OTU taxonomic table (OTU table) for the final analysis was ultimately created (supplemental table).

\section{Data Normalization and Statistical Analysis}

Venn diagrams were generated with R software to illustrate the unique and the shared OTUs among sampling sites [15]. The annotation ratio on each classification (phylum, class, order, family, and genus) rank was calculated to generate relative composition of each sample. According to the relative abundance of species at each classification, $\mathrm{R}$ software was applied to illustrate the histogram and heat map.

For a-diversity and $\beta$-diversity analyses, OTU tables were rarefied at 71000 tags from 16S rRNA and at 7830 tags from ITS tags per sample. a-diversity indices, including Chao1, Observed Species, Dominance, PD whole tree, Shannon and Simpson were calculated with QIIME (V1.9.1) [40] to analyze the complexity of species diversity. Among them, Chao1 was applied to identify community richness, while Shannon and Simpson were applied to identify community diversity. Bray-Curtis, weighted and unweighted unifrac beta diversity indexes were calculated by QIIME software, and accordingly, nonmetric multidimensional scaling (NDMS) analysis was performed and visualized by the vegan package of $\mathrm{R}$ software to further illustrate the $\beta$-diversity of soil microbial structure. Three non-parametric analyses (analysis of similarity (ANOSIM), non-parametric multivariate analysis of variance (Adonis) using distance matrices, and a multiresponse permutation procedure (MRPP)) were performed by R software based on the OTU table to evaluate the extent of differences among groups and whether the differences were significant $(P<0.05)$. A linear discriminant analysis (LDA) effect size (LEfSe) algorithm [41] was applied to identify the taxa in different abundances (biomarker, the threshold of the LDA score was set to 2) among the three sampling sites. To analyze the relationships among different microorganisms intra-kingdom network analysis was conducted using the 40 most abundant bacterial and fungal genera, and inter-kingdom network analysis was conducted using different microbial families. Highly significant positive $(R>0.6, P<0.05)$ and negative $(R>-0.6, P<0.05)$ Spearman correlations were screened out and co-occurrence patterns were visualized as networks using Cytoscape version 3.7.1 (https://cytoscape.org/) [42].

\section{Abbreviations}

operational taxonomic units was abbreviated as "OTUs"; nonmetric multidimensional scaling was abbreviated as "NDMS"; analysis of similarity was abbreviated as "ANOSIM"; non-parametric multivariate analysis of variance was abbreviated as "Adonis"; multiresponse permutation procedure was abbreviated as "MRPP"; linear discriminant analysis was abbreviated as "LDA".

\section{Declarations}

Ethics approval and consent to participate Not applicable. Animal experiments

related ethics procedures were not involved in this study; the samples used in this study were seasonal, natural, renewable resources. 
Consent for publication All authors approved the final manuscript and consented for publication.

Availability of data and materials All data generated or analyzed in study are included in this article and the supplementary information files.

Funding This work was jointly funded by the Natural Science Foundation of Guangdong Province (No. 2018A030313094, 2020A151501457), the National Natural Science Foundation of China (No. 81303155), "Group-type" Special Supporting Project for Educational Talents in Universities (4SG19057G), and the Project for Young Innovative Talents in Ordinary Higher University of Guangdong Province (No. 2018GkQNCX050).

Competing Interests No potential conflict of interest was reported by the authors.

Authors' contributions L.G. and H. T. conceived and designed the experiments; L.G. and Z.M. performed the experiments; Q.Z., X.G. and Y.H. analyzed the data; L.G. and X.L. wrote the paper.

All authors have read and approved the manuscript.

Acknowledgements Not applicable.

\section{References}

1. Zhang, Y.J.; Li, E.W.; Wang, C.S.; Li, Y.L.; Liu, X.Z. Ophiocordyceps sinensis, the flagship fungus of China: Terminology, life strategy and ecology. Mycology 2012;3: 2-

2. Xia, E.H.; Yang, D.R.; Jiang, J.J.; Zhang, Q.J.; Liu, Y.; Liu, Y.L.; Zhang, Y.; Zhang, H.B.; Shi, C.; Tong, Y.; Kim, C.; Chen, H.; Peng, Y.Q.; Yu, Y.; Zhang, W.; Eichler, E.E.; Gao, L.Z. The caterpillar fungus, Ophiocordyceps sinensis, genome provides insights into highland adaptation of fungal pathogenicity. Sci. Rep. 2017;7:

3. Cheng, R.L.; Yu, Y.X.; Liu, L.X.; Zhang, C.X.; Fang, C.X. A draft genome of the ghost moth, Thitarodes (Hepialus) sp., a medicinal caterpillar fungus. Insect Sci. 2016;23(2):326-

4. Lo, H.C.; Hsieh, C.; Lin, F.Y.; Hsu, T.H. A systematic review of the mysterious caterpillar fungus Ophiocordyceps sinensis in Dong-Chong-Xia-Cao (Dōng Chóng Xià Căo) and related bioactive ingredients. J. Tradit. Complement. Med. 2013;3(1):16-

5. Guo, L.X.; Zhang, G.W.; Wang, J.T.; Zhong, Y.P.; Huang, Z.G. Determination of arsenic species in Ophiocordyceps sinensis from major habitats in China by HPLC-ICP-MS and the edible hazard assessment. Molecules 2018;23:

6. Wang, X.L.; Yao, Y.J. Host insect species of Ophiocordyceps sinensis: a review. Zookeys 2011;127(127):43-

7. Li, Y.; Wang, X.L.; Jiao, L. A survey of the geographic distribution of Ophiocordyceps sinensis. J Microbiol 2011;49(6):913-

8. Shrestha, U.B.; Bawa, K.S. Impact of climate change on potential distribution of Chinese caterpillar fungus (Ophiocordyceps sinensis) in Nepal Himalaya. PLoS One 2014;9:

9. He, J. Harvest and trade of caterpillar mushroom (Ophiocordyceps sinensis) and the implications for sustainable use in the Tibet region of southwest China. J Ethnopharmacology 2018;221:86-

10. Li, X.; Liu, Q.; Li, W.J.; Li, Q.P.; Qian, Z.G.; Liu, X.Z.; Dong, C.Y. A breakthrough in the artificial cultivation of Chinese Cordyceps on a large-scale and its impact on science, the economy, and industry. Rev. Biotechnol. 2019;39:181-191. 
11. Guo, L.X.; Hong, Y.H.; Zhou, Q.Z.; Zhu, Q.; Xu, X.M.; Wang, J. Fungus-larva relation in the formation of Cordyceps sinensis as revealed by stable carbon isotope analysis. Sci. Rep. 2017;7:7789.

12. Shao, J.L.; Lai, B.; Jiang, W.; Wang, J.T.; Hong, Y.H.; Chen, F.B.; Tan, S.Q.; Guo, L.X. Diversity and cooccurrence patterns of soil bacterial and fungal communities of Chinese Cordyceps habitats at Shergyla Mountain, Tibet: Implications for the occurrence. Microorganisms 2019; 7:

13. Guo, L.; Xu, X.M.; Liang, F.R.; Yuan, J.P.; Peng, J.; Wu, C.F.; Wang, J.H. Morphological observations and fatty acid composition of indoor-cultivated Cordyceps sinensis at a high-altitude laboratory on Sejila Mountain, Tibet. PLoS One 2015;10(5):e0126095.

14. Liang, Y.; Hong, Y.; Mai, Z.; Zhu, Q.; Guo, L. Internal and external microbial community of the Thitarodes moth, the host of Ophiocordyceps sinensis. Microorganisms 2019;7:517.

15. Shade, A.; Handelsman, J. Beyond the Venn diagram: the hunt for a core microbiome. Microbiol. 2012; 14(1): 4-12.

16. Zheng, P.; Xia, Y.; Zhang, S.; Wang, C. Genetics of Cordyceps and related fungi. Appl. Biotechnol. 2013; 97 : 2797-2804.

17. Wang, J.; Wei, H.Y. Anatomy of internal structure of the larva of Hapialus hunanensis Chu et Wang (Lepidoptera, Hepialidae). Acta. Agric. Univ. Jiangxi 1997; 19: 5-8.

18. Chen; Gao, Z.X.; Yu, H. Observation on egg's feature and chorionic ultrastructure of swiftmoth (Hepialus oblifurcus Chu et Wang). Acta. Agric. Univ. Zhejiang 1991; 39: 379-383.

19. Yue, K.; Ye, M.; Lin, X. The artificial cultivation of medicinal Caterpillar Fungus, Ophiocordyceps sinensis (Ascomycetes): a review. International Journal of Medicinal Mushrooms 2013; 15(5): 425.

20. Zhang, Y.; Zhang, S.; Wang, M.; Bai, F.; Liu, X. High diversity of the fungal community structure in naturallyoccurring Ophiocordyceps sinensis. Plos One 2010; 5: e15570.

21. Ko, Y.F.; Liau, J.C.; Lee, C.S.; Chiu, C.Y.; Martel, J.; Lin, C.S.; Tseng, S.F.; Ojcius, D.M.; Lu, C.C.; Lai, H.C.; Young, J.D. Isolation, culture and characterization of Hirsutella sinensis mycelium from caterpillar fungus fruiting body. Plos One 2017; 12: e0168734.

22. Li, Y.L.; Yao, Y.S.; Zhang, Z.H.; Liu, X.; Xu, H.F.; Ma, S.L.; Wu, Z.W.; Zhu, J.S. Synergy of fungal complexes isolated from the intestines of Hepialus lagii larvae in increasing infection potency. J Fungal Res 2016; 14(2): 96112.

23. Liang, Z.Q.; Han, Y.F.; Liang, J.D.; Dong, X.; Du, W. Issues of concern in the studies of Ophiocordyceps sinensis. Microbiol 2010; 37: 1692-1697.

24. Zhang Y. 2016. Studies on the relationship between the strains related to Ophiocordyceps and Hirsutella Dissertation for the master Degree. Lanzhou Jiaotong University.

25. de Menezes, A.B.; Richardson, A.E.; Thrall, P.H. Linking fungal-bacterial co-occurrences to soil ecosystem Curr. Opin. Microbiol. 2017, 37, 135-141.

26. Faust, K.; Lima-Mendez, G.; Lerat, J.S.; Sathirapongsasuti, J.F.; Knight, R.; Huttenhower, C.; Lenaerts, T.; Raes, J. Cross-biome comparison of microbial association networks. Front. Microbiol. 2015, 6, 1200.

27. Sung, G.H.; Hywel-Jones, N.L.; Sung, J.M.; Luangsa-Ard, J.J.; Shrestha, B.; Spatafora, J.W. Phylogenetic classification of Cordyceps and the Clavicipitaceous Studies Mycol. 2007;57:5-59.

28. Eberhard, W.; Pacheco-Esquivel, J.; Carrasco-Rueda, F.; Christopher, Y.; Gonzalez, C.; Ramos, D.; Urbina, H.; Blackwell, M. Zombie bugs? The fungus Purpureocillium lilacinum may manipulate the behavior of its host 
bug Edessa rufomarginata. Mycologia 2014; 106(6): 10651072.

29. Zhong, X.; Peng, Q.Y.; Li, S.S.; Chen, H.; Sun, H.X.; Zhang, G.R.; Liu, X. Detection of Ophiocordyceps sinensis in the roots of plants in alpine meadows by nested-touchdown polymerase chain reaction. Fungal Biol. 2014; 118: 359-363.

30. Goto, S.; Anbutsu, H.; Fukatsu, T. Asymmetrical interactions between Wolbachia and Spiroplasma endosymbionts coexisting in the same insect host. Environ. Microbiol. 2006; 72(7): 4805-4810.

31. Werren, J.H.; Baldo, L.; Clark, M.E. Wolbachia: master manipulators of invertebrate biology. Nature Rev 2008; 6(10): 741-751.

32. Teixeira, L.; Ferreira, Á.; Ashburner, M. The bacterial symbiont Wolbachia induces resistance to RNA viral infections in Drosophila melanogaster. PLoS Biol 2008; 6(12): e2.

33. Zug, R.; Peter, H. Wolbachia and the insect immune system: what reactive oxygen species can tell us about the mechanisms of Wolbachia-host interactions. Front Microbio, 2015; 6:1201.

34. Min, Q.; Cheng, S.; Xi, J.; Xin, T.; Xia, B.; Zou, Z. Differential expression patterns of two delta-9-acyl-CoA desaturases in Thitarodes pui (Lepidoptera: Hepialidae) during different seasons and cold exposure. and Evol. 2017;7(6):1909-1918.

35. Bolger, A.M.; Lohse, M.; Usadel, B. Trimmomatic: a flexible trimmer for Illumina sequence data. Bioinformatics 2014;30(15): 2114-

36. Magoč, T:; Salzberg, S. FLASH: fast length adjustment of short reads to improve genome assemblies. Bioinformatics 2011;27(21):2957-2963.

37. Schloss, P.D.; Westcott, S.L.; Ryabin, T.; Hall, J.R.; Hartmann, M.; Hollister, E.B.; Lesniewski, R.A.; Oakley, B.B.; Parks, D.H.; Robinson, C.J.; Sahl, J.W.; Stres, B.; Thallinger, G.G.; Van Horn, D.J.; Weber, C.F. Introducing mothur: open-source, platform-independent, community-supported software for describing and comparing microbial communities. Appl. Environ. Microbiol. 2009,75(23):

38. Edgar, R.C. Search and clustering orders of magnitude faster than BLAST. Bioinformatics 2010; 26(19): 2460-2461.

39. Tindall, B.J.; Rossello-Mora, R.; Busse, H. J.; Ludwig, W.; Kampfer, P. Notes on the characterization of prokaryote strains for taxonomic purposes. Int J Syst Evol Micr 2010; 60: 249-266.

40. Caporaso, J.G.; Kuczynski, J.; Stombaugh, J.; Bittinger, K.; Knight, R. QIIME allows analysis of highthroughput community sequencing data. Methods 2010; 7: 335-336.

41. Segata, N.; Izard, J.; Waldron, L.; Gevers, D.; Miropolsky, L.; Garrett, W.S.; Huttenhower, C. Metagenomic biomarker discovery and explanation. Genome Biol. 2011; 12:

42. Shannon, P.; Markiel, A.; Ozier, O.; Baliga, N.S.; Wang, J.T.; Ramage, D.; Amin, N.; Schwikowski, B.; Ideker, T. Cytoscape: A software environment for integrated models of biomolecular interaction networks. Genome Res. 2003, 13, 2498-2504.

\section{Tables}

Table 1. Diversity indices of microbial communities in fertilized Thitarodes eggs based on 16S rRNA and internal transcribed spacer (ITS) genes analysis. 


\begin{tabular}{cccccccc}
\hline Classified & Sample site & Chao1 & Observed Species & PD whole tree & Shannon & Simpson & Dominance \\
\hline Bacteria & Site A & $1238 \pm 124$ & $549.2 \pm 110.868$ & $31.6 \pm 5.273$ & $4.8 \pm 0.7$ & $0.860 \pm 0.077$ & $0.141 \pm 0.077$ \\
& Site B & $1553 \pm 876$ & $1026 \pm 884.982$ & $51.2 \pm 37.218$ & $1.6 \pm 1.3^{*}$ & $0.229 \pm 0.158^{*}$ & $0.771 \pm 0.159^{*}$ \\
& Site C & $1145 \pm 82$ & $493.4 \pm 33.396$ & $26.8 \pm 2.168$ & $1.4 \pm 0.4^{*}$ & $0.316 \pm 0.133^{*}$ & $0.684 \pm 0.133^{*}$ \\
& & & & & & & \\
Fungi & Site A & $656 \pm 242$ & $355 \pm 137.744$ & $169.8 \pm 40.339$ & $5.0 \pm 1.0$ & $0.885 \pm 0.117$ & $0.115 \pm 0.117$ \\
& & & & & & & \\
& Site B & $768 \pm 136$ & $472.2 \pm 162.2$ & $121.8 \pm 36.044$ & $6.1 \pm 1.2$ & $0.941 \pm 0.039$ & $0.059 \pm 0.039$ \\
& & & & & & & \\
& Site C & $610 \pm 149$ & $386 \pm 107.264$ & $211.2 \pm 14.772 \#$ & $5.1 \pm 0.6$ & $0.887 \pm 0.028 \#$ & $0.113 \pm 0.028 \#$ \\
\hline
\end{tabular}

${ }^{*} P<0.05$ compared to site A; \# $P<0.05$ compared to site $\mathrm{B}$. OTU, operational taxonomic unit.

Table 2. Dissimilarity comparison of microbial community structure of fertilized Thitarodes eggs among all sampling sites. ANOSIM, analysis of similarities; MRPP, multiresponse permutation procedure.

\begin{tabular}{|c|c|c|c|c|c|c|c|c|c|}
\hline & \multicolumn{2}{|c|}{ ANOSIM } & \multicolumn{3}{|c|}{ Adonis } & \multicolumn{4}{|c|}{ MRPP } \\
\hline & $R$ & $P$ & $F$ & $R^{2}$ & $P$ & $\begin{array}{l}\text { Observed } \\
\text { Delta (6) }\end{array}$ & $\begin{array}{l}\text { Expected } \\
\text { Delta (6) }\end{array}$ & $\begin{array}{c}\text { Effect } \\
\text { size (A) }\end{array}$ & $P$ \\
\hline \multirow[t]{2}{*}{ Bacteria } & 0.605 & & 19.34 & 0.763 & 0.001 & 0.300 & & & \\
\hline & & 0.001 & & & & & 0.572 & 0.476 & 0.001 \\
\hline \multirow[t]{2}{*}{ Fungi } & & & 1.915 & 0.242 & 0.027 & 0.722 & 0.772 & 0.064 & 0.026 \\
\hline & 0.270 & 0.018 & & & & & & & \\
\hline
\end{tabular}

Table 3. Intra-kingdom and inter-kingdom analysis of soil microbial communities.

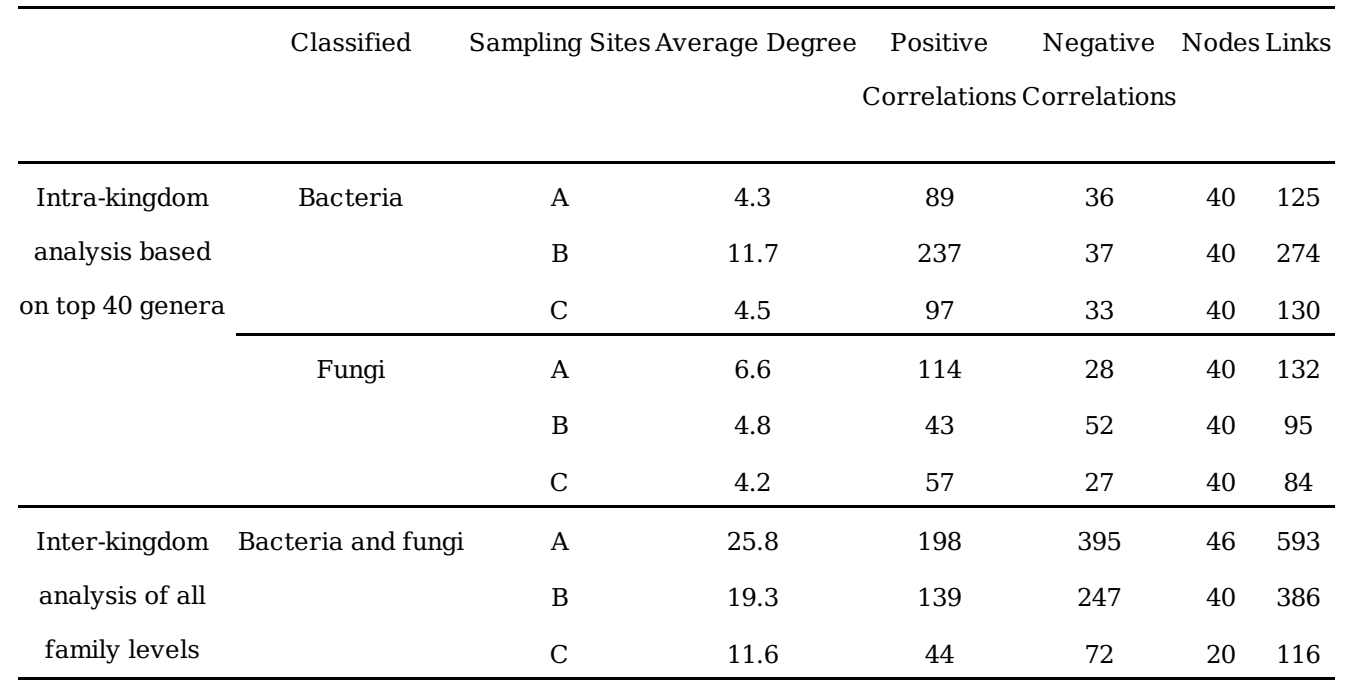




\section{Supplementary Materials}

Supplemental table: OTU table and relative compositions of bacterial communities at the phylum, class, order, family, and genus level.

\section{Figures}

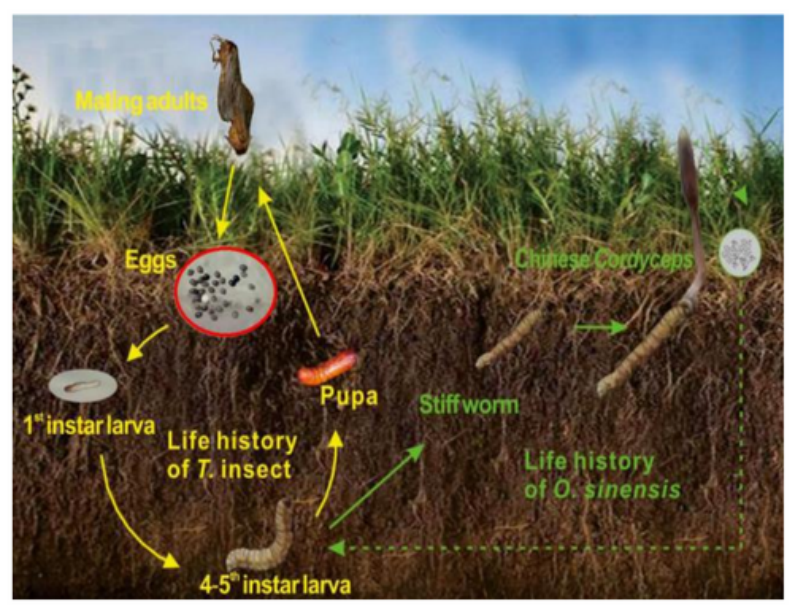

(a)

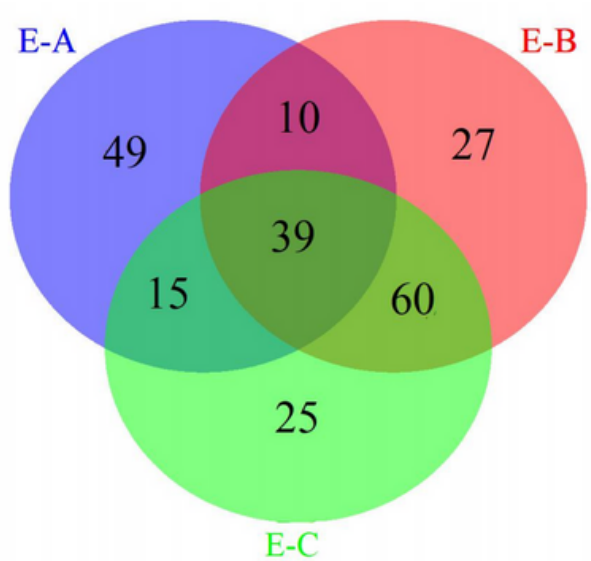

(b)

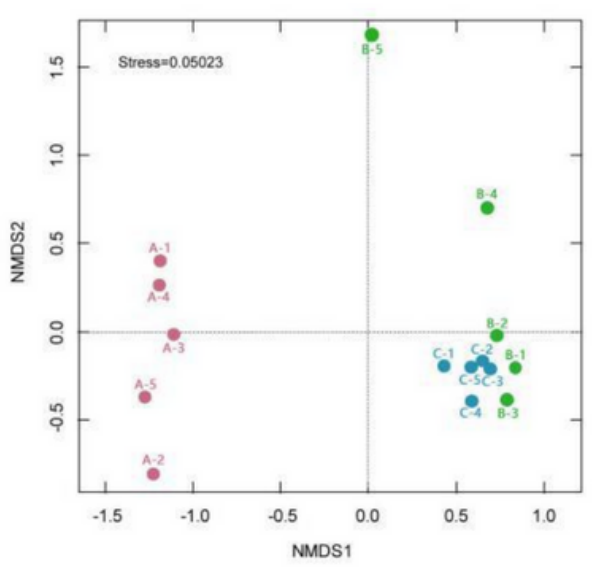

(d)

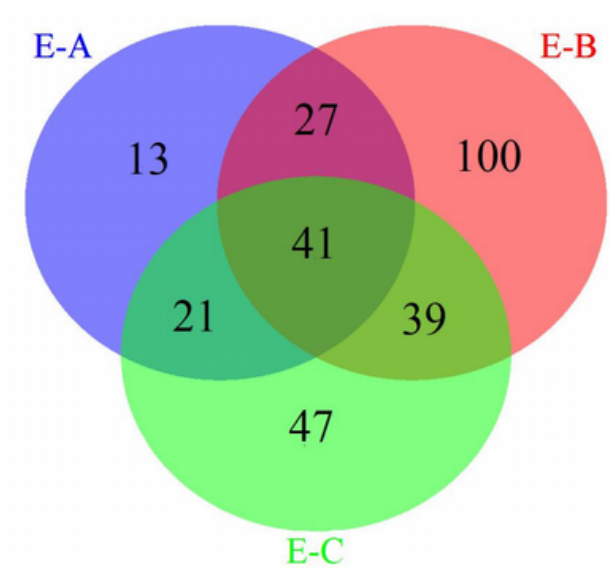

(c)

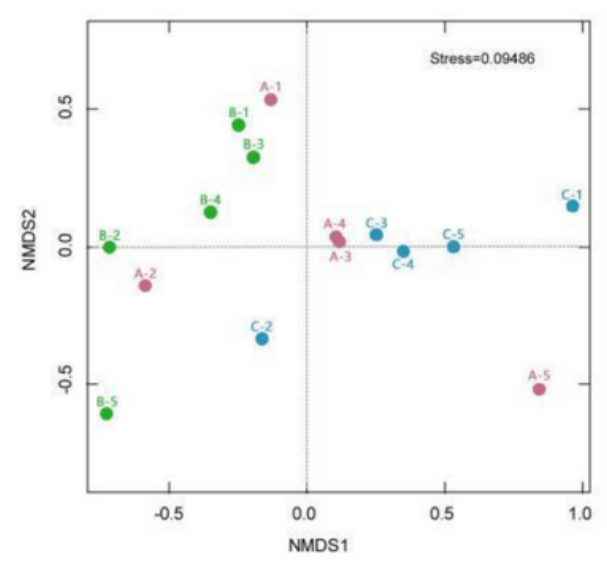

(e) 


\section{Figure 1}

Life history of Ophiocordyceps sinensis and Thitarodes host (a), Venn diagrams and nonmetric multidimensional scaling of bacterial ( $b$ and $d$ ) and fungal ( $c$ and e) communities in fertilized Thitarodes eggs. (a) this figure is modified according to the previous study [5], red oval highlights the research sample for this study; (b) and (c), Venn diagrams depicting the unique and shared OTUs among the samples, and the detailed taxonomic information of the shared OTUs were supplemented in supplemental material. (d) and (e), nonmetric multidimensional scaling based on Euclidean distance plot. $A, B$, and $C$ present different sampling sites with high, low, and null Chinese cordyceps, respectively.

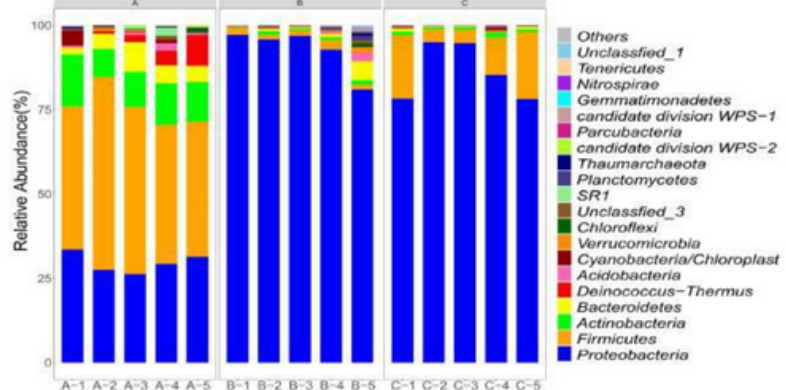

(a)

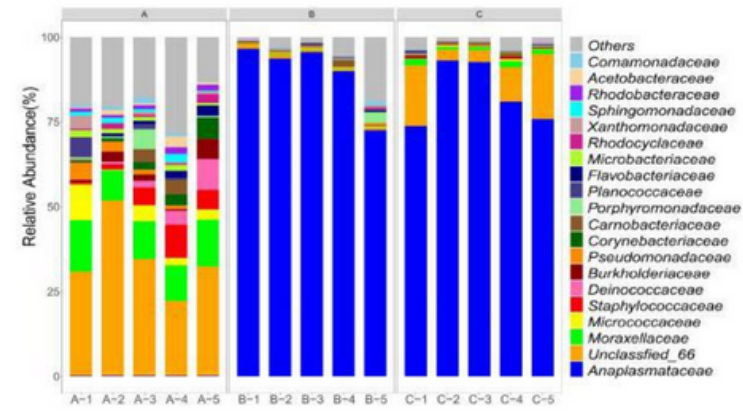

(c)

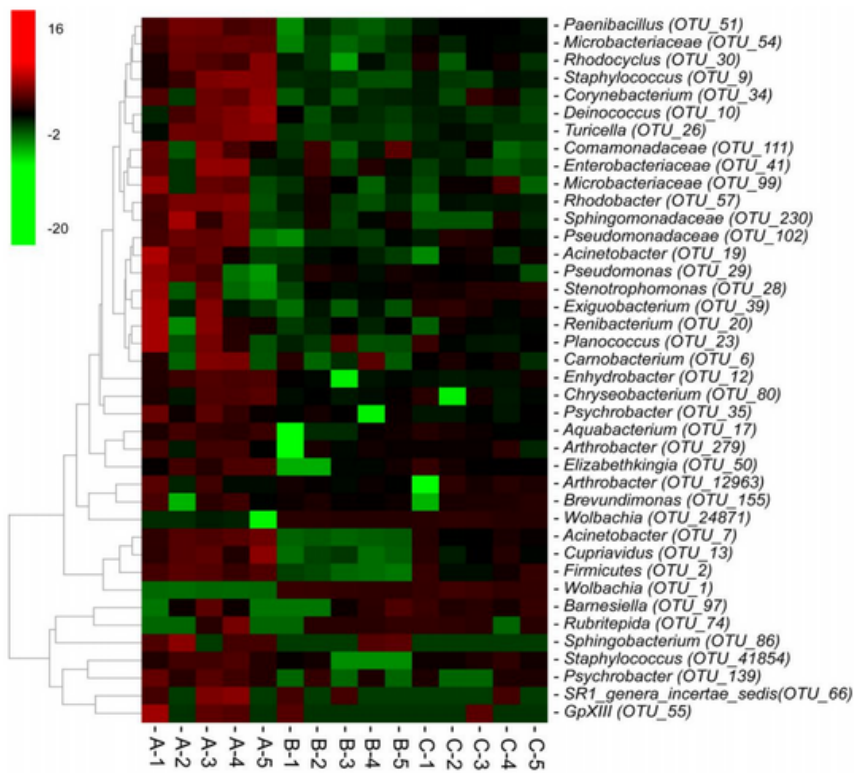

(e)

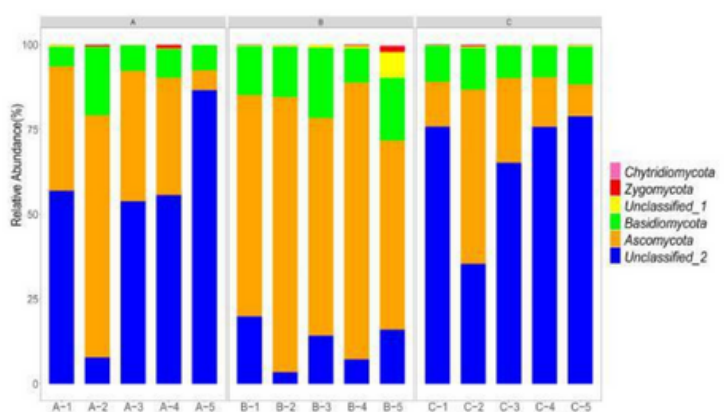

(b)

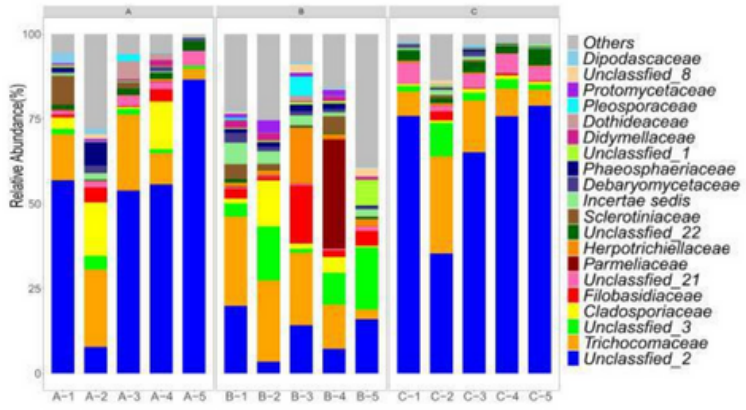

(d)

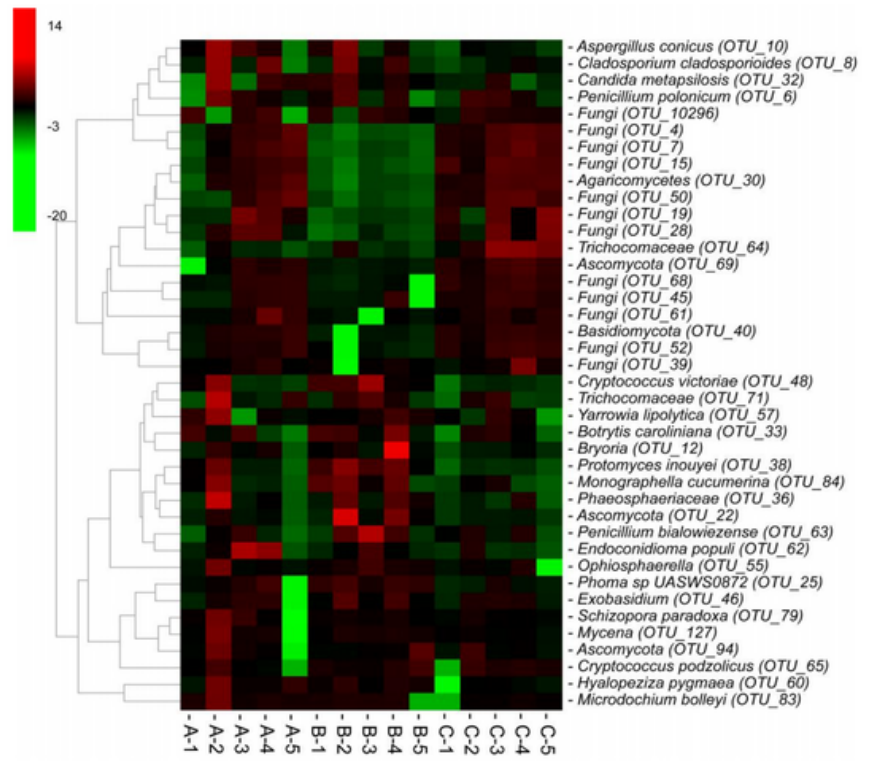

(f) 
Figure 2

Relative abundance of microbial phyla ( $a$ and b) and families (c and d), and Heatmaps of top 40 bacterial (e) and fungal (f) OTUs in the fertilized Thitarodes eggs from three sampling sites. "Others" includes phyla with less than $0.1 \%$ of relative abundance. $A, B$, and $C$ present different sampling sites with high, low, and null Chinese cordyceps, respectively.

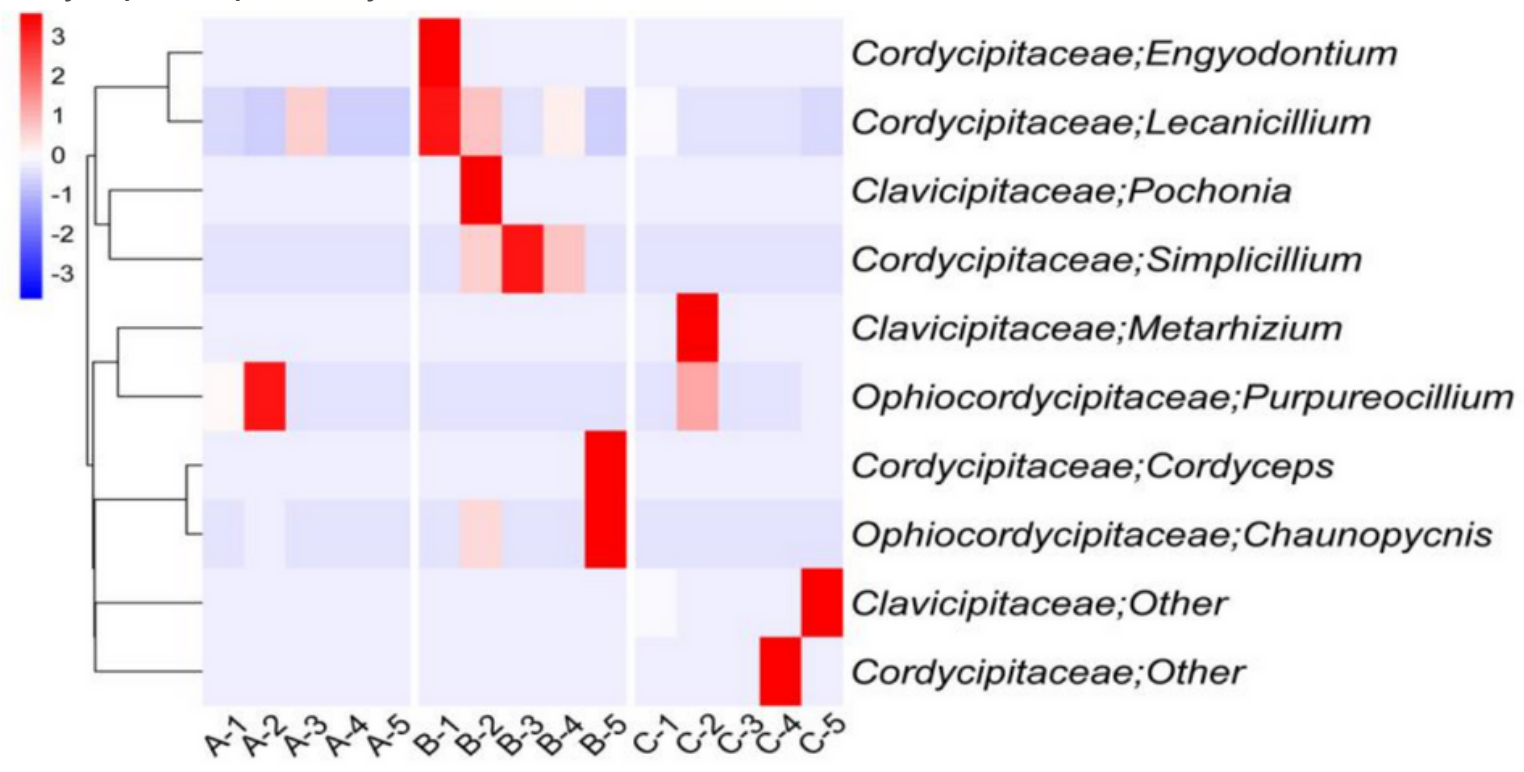

(a)

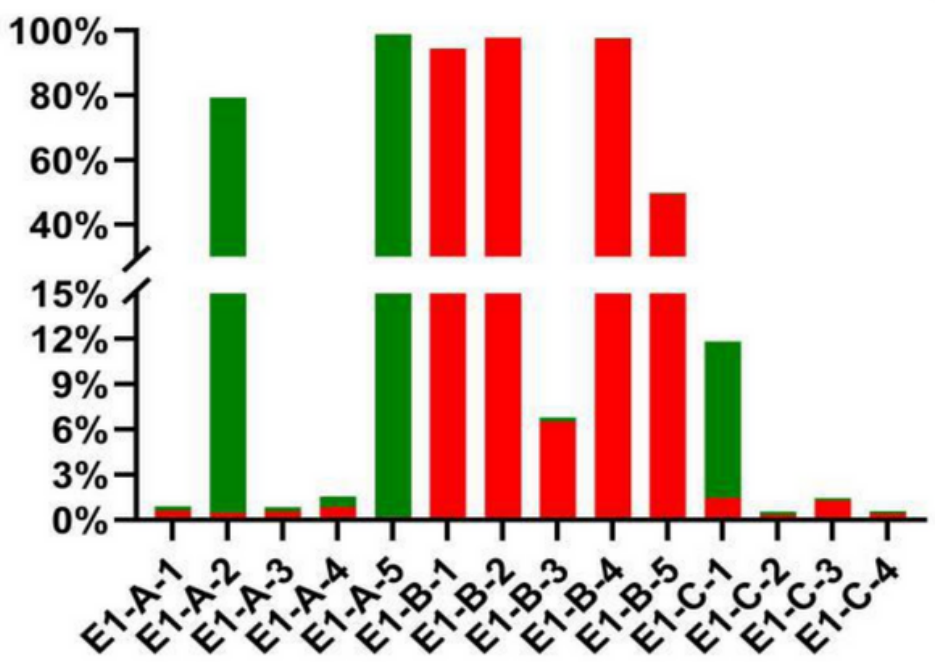

(b)

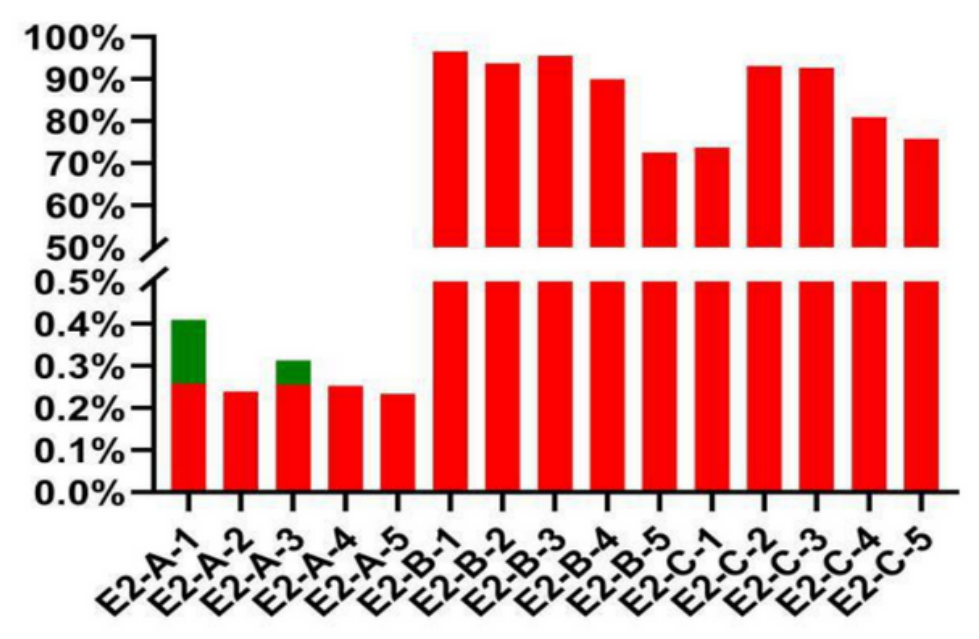

(c)

Figure 3

The relative abundances of Cordyceps-related fungal genera illustrated by heatmap (a), and the relative abundances of OTU1 (Wolbachia) and OTU4 (Spiroplasma) in unfertilized eggs (E1, b) and fertilized eggs (E2, C). A, B, and C present different sampling sites with high, low, and null Chinese cordyceps, respectively. The data of unfertilized eggs were cited from our previous study [14].

要

Figure 4 
Differentially abundant microbial taxa in three sampling sites illustrated using linear discriminant analysis (LDA) effect size analysis and cladograms for bacteria $(a, c)$ and fungi $(b, d)$. Red, green,and blue represents taxa enriched in sites $A, B$ and $C$, respectively. Microbial taxa were determined by LDA with a significant threshold over 2.

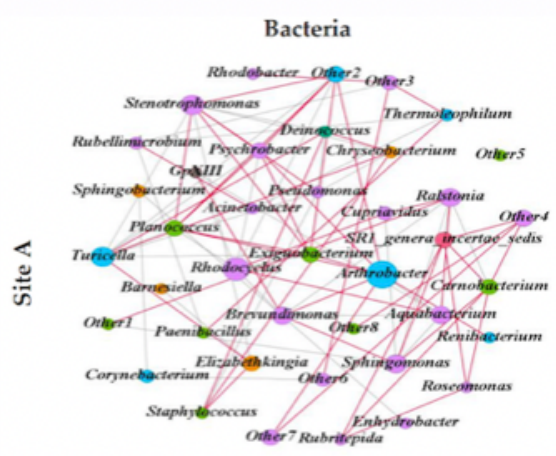

(a)

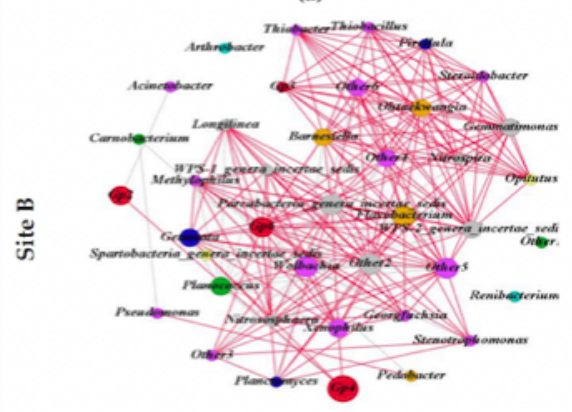

(c)

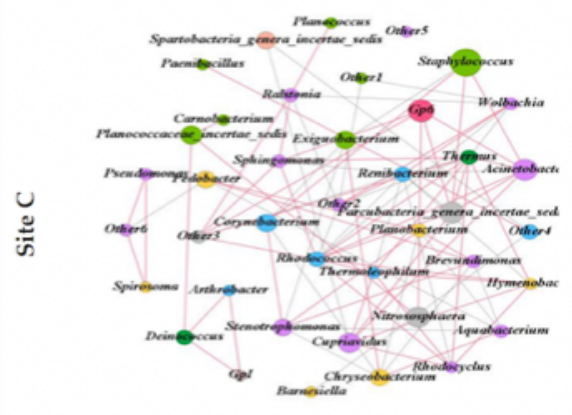

(e)

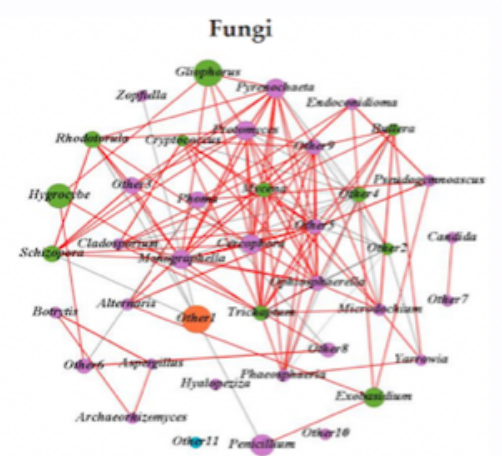

(b)

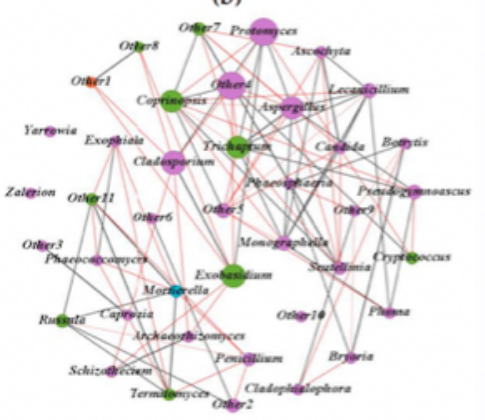

(d)

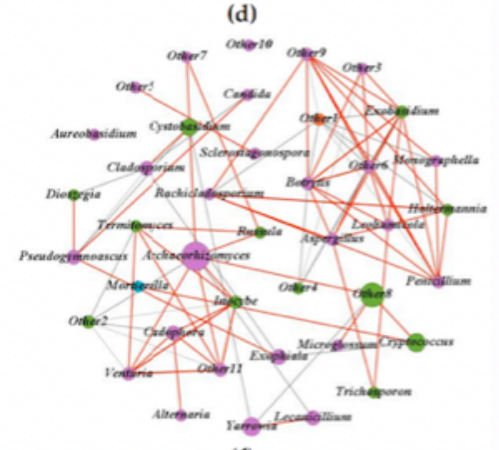

(f)

(Bacteria: Proteobacteria, Firmicutes, Ectinobacteria, Bacteroidetes, Eyanobacteria,

SR1, and Deinococcus-Thermus; Fungi: -Ascomycota, Basidiomycota, Dther, and Mucoromycota)

\section{Figure 5}

Intra-kingdom network of 40 most abundant co-occurring bacterial and fungal genera based on correlation analysis. Connecting lines represent significant correlation. Highly significant positive $(R>0.6, P<0.05)$ and 
negative $(R>\otimes 0.6, P<0.05)$ Spearman correlations are represented as red and gray lines. The size of each node is proportional to the degree, and nodes are colored by taxonomy

\section{Figure 6}

Inter-kingdom network of differentially abundant fungal and bacterial families at sites A (a), B (b), and C (c). Highly significant positive $(R>0.80, F D R<0.05)$ and negative $(R<-0.80$, FDR $<0.05)$ Spearman correlations are represented by red and gray lines. The size of each node is proportional to the degree.

\section{Supplementary Files}

This is a list of supplementary files associated with this preprint. Click to download.

- STableall.xIsx 